\title{
Ion dynamics in post harvest saline soil influenced by organic amendment and moisture level
}

\author{
S. Akter and H. R. Khan
}

Department of Soil, Water and Environment, University of Dhaka, Dhaka 1000, Bangladesh

Received: 31 March 2021

Revised: 21 June 2021

Accepted: 28 June 2021

DOI: https://doi.org/10.3329/bjsir.v56i3.55966

\begin{abstract}
Organic amendments might be effective and sustainable in the amelioration of saline soil if proper management put in place. Accordingly, subsequent pot and field experiments were conducted in a saline soil to determine the effects of moisture levels and organic amendments on cation exchange capacity (CEC), ion dynamics under rice cultivation. The increment of CEC of the studied post harvest soils was significant, except for the moist condition under field experiment. There were significant variations in ion dynamics among the treatments under both the experiments at saturated soil conditions. Exchangeable $\mathrm{Na}^{+}$contents decreased and $\mathrm{K}^{+}$contents increased significantly with the increased rates of treatments. Exchangeable $\mathrm{Ca}^{2+}$ and $\mathrm{Mg}^{2+}$ contents of soil followed almost the similar trends as that exhibited by $\mathrm{K}^{+}$but not significant. Among the anions, chloride decreased significantly under both the experiments while sulfate and bicarbonate contents increased by the applied treatments. This might be due to the inherent consequences of organic amendments on these soil properties.
\end{abstract}

Keywords: CEC; Ions; Saline soil; Organic amendments and Moisture levels

\section{Introduction}

Interpretations in the recent past indicated that due to increasing degree of salinity of some areas and expansion of salt affected area as a cause of further intrusion of saline water, normal crop production becomes more restricted (Akter et al., 2017). Saline soils are an important natural resource but the area of degraded saline soils worldwide has rapidly increased due to climate change and limited rainfall, which poses a great challenge to global food security (Yupeng et al., 2018). This problem may be solved through a targeted remediation program of such soils. Ions that contribute to soil salinity include $\mathrm{Cl}^{-}, \mathrm{SO}_{4}{ }^{2-}, \mathrm{HCO}_{3}^{-}, \mathrm{Na}^{+}, \mathrm{Ca}^{2+}, \mathrm{Mg}^{2+}$, and, rarely, $\mathrm{NO}_{3}^{-}$or $\mathrm{K}^{+}$. Salinization affects the metabolism of the organisms present in the soil, drastically reducing soil fertility and increasing water proofing of the deeper layers (Rahman et al., 2014).

Salinity decreases the osmotic potential of the soil, which leads to decreased turgor pressure in root cells and consequently water loss (Julkowska and Testerink, 2015). To avoid water loss, a first response of the plant is to close stomata and reduce transpiration at the cost of lower cell extension rate and growth. The maintenance of turgor is also facilitated by decreasing the osmotic potential in the roots, which is achieved by increasing the concentration of osmolytes in tissues. Osmotic adjustment is an essential plant response to salt stress, and can be achieved by the synthesis of organic compounds, or the accumulation of $\mathrm{Na}^{+}$and $\mathrm{Cl}^{-}$ in a cost-effective manner (Munns et al., 2016). In addition to the challenge of transporting water under salt stress, the plant has to deal with the salt ions $\left(\mathrm{Na}^{+}\right.$ and $\mathrm{Cl}^{-}$) that are taken up and that are toxic at high concentrations. A recent review examines the implications of keeping ion concentrations in shoots of plants are low (Munns et al., 2020). 
Salinity also affects soil chemical properties, such as $\mathrm{pH}$, cation exchange capacity (CEC), exchangeable sodium percentage (ESP), soil organic carbon, and alters the osmotic and matric potential of the soil solution. Most salt-affected soils are deficient in several nutrients; thus, more fertilizer applications may be required. In Bangladesh, out of about 1.689 million hectares of coastal land, 1.056 million hectares are affected by soil salinity of various degrees. About $50 \%$ of the coastal lands face different degrees of inundation, thus limiting their effective use (Islam, 2006). This situation is expected to become worse further because of the effects of climate change.

Application of organic amendments was reported to remediate saline soils, alleviate salinity and sodicity stress on crops (Seleiman and Kheir, 2018). Organic amendments could improve soil properties by accelerating leaching of sodium and other salts and reducing exchangeable sodium percentage (ESP). Organic matter decomposition and plant root action also help dissolve the calcium compounds found in most soils, thus promoting reclamation of saline soil (Gupta and Gupta, 1987). Organic amendments improve physical, chemical and biological properties of soils under saline conditions (Raafat and Tharwat, 2011). In any case, the application of organic amendments could help to increase the cation exchange capacity (CEC) and ion adsorption with all positive/negative consequences, and therefore reduce the soluble salt concentration in the soil solution. In fact, organic matter generally is considered one of the most important contributors to the CEC in the soil, determining the sign and magnitude of net surface charge of soil particles and enhancing the retention of soil salts (Oorts et al., 2003). Therefore, the present research work had been undertaken to investigate the changes in saline soil CEC, exchangeable cations $\left(\mathrm{Na}^{+}, \mathrm{K}^{+}, \mathrm{Ca}^{2+}\right.$ and $\left.\mathrm{Mg}^{2+}\right)$ and water-soluble anions $\left(\mathrm{Cl}^{-}, \mathrm{SO}_{4}{ }^{2-}\right.$ and $\left.\mathrm{HCO}_{3}^{-}\right)$ under rice cultivation as influenced by organic amendments like rice hull, rice straw, sawdust and two moisture levels on pot and field experiments.

\section{Materials and methods}

The experiments were conducted under field and pot conditions during Boro and Aus seasons, respectively. Considering climatic condition and growing season, the field experiment was conducted at Musulliabad (Lata Chapli), Kalapara, Patuakhali, Bangladesh during January to June, 2017 with a T. Boro rice cultivar (BRRI dhan 64). Subsequently, a pot experiment was also conducted with a $\mathrm{T}$. Aus rice cultivar (BRRI dhan 48) in the premises of the
Department of Soil, Water and Environment, University of Dhaka during May to August, 2017 in order to compare the effects of treatments under open (field) and closed (pot) systems. The soil used in the pot experiment was same to the field soil. Physico-chemical characteristics of initial soil (Table I) were determined by standard methods.

Rice hull (RH), rice straw (RS) and sawdust (SD) were used as indigenous organic amendments for the studied soil. The experiments were done with rice hull ( 3 levels) $\times$ rice straw (3 levels) $\times$ sawdust ( 3 levels) having 3 replications (considered within the plot and pot) under 2 moisture levels (moist: $80 \%$ moisture and saturated: $>100 \%$ moisture). Total number of treatments was 27 (for each moisture levels; Table II). Basal doses of $\mathrm{N}, \mathrm{P}_{2} \mathrm{O}_{5}$ and $\mathrm{K}_{2} \mathrm{O}$ were applied at the rate of 120, 60 and $80 \mathrm{~kg} \mathrm{ha}^{-1}$ as Urea, TSP and MoP, respectively. The whole TSP, MoP and half of the urea were applied during soil preparation. The remaining urea was top dressed in two splits, at active tillering and panicle initiation stage.

Seedlings of BRRI dhan 64 were collected from the local experienced farmers. Thirty-days-old seedlings of BRRI dhan 64 were transplanted at the rate of 3 seedlings per hill. The hill to hill and row to row distances were 18 and $22 \mathrm{~cm}$, respectively. Seedlings of BRRI dhan 48 were collected through the courtesy of BRRI (Bangladesh Rice Research Institute), Joydebpur, Gazipur. About 25 days-old seedlings of BRRI dhan 48 were transplanted at the rate of 3 seedlings per hill and 3 hills in each pot by arranging in a triangular grid.

In the case of moist condition: $80 \%$ water content seemed to be optimum for the survival of rice plant and did not allow standing water. But in the case of saturated condition: more than $100 \%$ water was maintained during irrigation throughout the growing period. Intercultural operations were performed as required.

CEC, exchangeable cations $\left(\mathrm{Na}^{+}, \mathrm{K}^{+}, \mathrm{Ca}^{2+}\right.$ and $\left.\mathrm{Mg}^{2+}\right)$ and water-soluble anions $\left(\mathrm{Cl}^{-}, \mathrm{SO}_{4}{ }^{2-}\right.$ and $\left.\mathrm{HCO}_{3}^{-}\right)$of the soils were analyzed after harvesting the crop at maturity. MS. Excel and Stata 14 computer programs were used to analyze the experimental data. The significance of different treatments was assessed by calculating CV (Coefficient of Variation) and Tukey's Range Test of the experimental results done for the interpretation.

\section{Results and discussion}

Dynamics in cation exchange capacity (CEC)

CEC refers to the amounts of negative charges available on the surface of soil particles. It gives an indication of the 
Table I. Physico-chemical properties of the studied soil on oven dry basis (After Akter et al., 2017)

\begin{tabular}{|c|c|}
\hline Properties & Values \\
\hline Particle density $\left(\mathrm{g} \mathrm{cm}^{-3}\right)$ & 2.53 \\
\hline Bulk density $\left(\mathrm{g} \mathrm{cm}^{-3}\right)$ & 1.37 \\
\hline Porosity $(\%)$ & 45.81 \\
\hline Moisture content (\%; Black, 1965) & 3.31 \\
\hline Textural class (Hydrometer method; Piper, 1966) & Clay loam \\
\hline pH (1:2.5; Jackson, 1973) & 6.90 \\
\hline EC (dS m ${ }^{-1}$; Saturation extract, 1:5; Richards, 1954) & 3.96 \\
\hline Organic carbon ( $\mathrm{g} \mathrm{kg}^{-1}$; Nelson and Sommers, 1982) & 7.80 \\
\hline Total Nitrogen ( $\mathrm{g} \mathrm{kg}^{-1} ;$ Jackson, 1973) & 0.60 \\
\hline Available Nitrogen ( $\mathrm{mg} \mathrm{kg}^{-1} ; 1 \mathrm{~N} \mathrm{KCl}$; Jackson, 1973) & 54.55 \\
\hline Available Phosphorus ( $\mathrm{mg} \mathrm{kg}^{-1}$; Olsenet al., 1954) & 12.58 \\
\hline Available Potassium (mg kg ${ }^{-1} ;$ Pratt, 1965) & 25.00 \\
\hline \multicolumn{2}{|l|}{ Exchangeable Cations ( $\left.\mathrm{c} \mathrm{mol} \mathrm{kg}^{-1} ; 1 \mathrm{~N} \mathrm{CH}_{3} \mathrm{COONH}_{4}\right)$} \\
\hline Sodium (flame photometer) & 3.91 \\
\hline Potassium (flame photometer) & 0.64 \\
\hline Calcium $\left(\mathrm{AAS}^{*}\right)$ & 1.87 \\
\hline Magnesium (AAS*) & 3.26 \\
\hline \multicolumn{2}{|l|}{ Water Soluble Anions ( $\mathrm{c} \mathrm{mol} \mathrm{kg}^{-1}$ ) } \\
\hline Chloride (0.005 N AgNO & 2.87 \\
\hline Sulphate $\left(\mathrm{BaCl}_{2} ;\right.$ Richards, 1954$)$ & 1.45 \\
\hline Bicarbonate $\left(0.05 \mathrm{~N} \mathrm{H}_{2} \mathrm{SO}_{4}\right.$; Richards, 1954) & 0.47 \\
\hline Carbonate $\left(0.05 \mathrm{~N} \mathrm{H}_{2} \mathrm{SO}_{4} ;\right.$ Richards, 1954) & ND* \\
\hline Cation Exchange Capacity (c mol kg-1; Black, 1965) & 18.67 \\
\hline Sodium Adsorption Ratio (Richards, 1954) & 7.72 \\
\hline Exchangeable Sodium Percentage (Richards, 1954) & 20.92 \\
\hline
\end{tabular}

$* \mathrm{ND}=$ Not in detectable range, $* \mathrm{AAS}=$ Atomic Absorption Spectrophotometer

Table II. Treatment combination of the experiment

\begin{tabular}{cccccc}
\hline \multicolumn{7}{c}{ Treatment } \\
\hline \multicolumn{1}{c}{ No. } & Denotation & No. Denotation & No. & Denotation \\
\hline $\mathrm{T}_{1}$ & $\mathrm{RH}_{0} \mathrm{RS}_{0} \mathrm{SD}_{0}$ & $\mathrm{~T}_{10}$ & $\mathrm{RH}_{4} \mathrm{RS}_{0} \mathrm{SD}_{0}$ & $\mathrm{~T}_{19}$ & $\mathrm{RH}_{8} \mathrm{RS}_{0} \mathrm{SD}_{0}$ \\
$\mathrm{~T}_{2}$ & $\mathrm{RH}_{0} \mathrm{RS}_{0} \mathrm{SD}_{4}$ & $\mathrm{~T}_{11}$ & $\mathrm{RH}_{4} \mathrm{RS}_{0} \mathrm{SD}_{4}$ & $\mathrm{~T}_{20}$ & $\mathrm{RH}_{8} \mathrm{RS}_{0} \mathrm{SD}_{4}$ \\
$\mathrm{~T}_{3}$ & $\mathrm{RH}_{0} \mathrm{RS}_{0} \mathrm{SD}_{8}$ & $\mathrm{~T}_{12}$ & $\mathrm{RH}_{4} \mathrm{RS}_{0} \mathrm{SD}_{8}$ & $\mathrm{~T}_{21}$ & $\mathrm{RH}_{8} \mathrm{RS}_{0} \mathrm{SD}_{8}$ \\
$\mathrm{~T}_{4}$ & $\mathrm{RH}_{0} \mathrm{RS}_{4} \mathrm{SD}_{0}$ & $\mathrm{~T}_{13}$ & $\mathrm{RH}_{4} \mathrm{RS}_{4} \mathrm{SD}_{0}$ & $\mathrm{~T}_{22}$ & $\mathrm{RH}_{8} \mathrm{RS}_{4} \mathrm{SD}_{0}$ \\
$\mathrm{~T}_{5}$ & $\mathrm{RH}_{0} \mathrm{RS}_{4} \mathrm{SD}_{4}$ & $\mathrm{~T}_{14}$ & $\mathrm{RH}_{4} \mathrm{RS}_{4} \mathrm{SD}_{4}$ & $\mathrm{~T}_{23}$ & $\mathrm{RH}_{8} \mathrm{RS}_{4} \mathrm{SD}_{4}$ \\
$\mathrm{~T}_{6}$ & $\mathrm{RH}_{0} \mathrm{RS}_{4} \mathrm{SD}_{8}$ & $\mathrm{~T}_{15}$ & $\mathrm{RH}_{4} \mathrm{RS}_{4} \mathrm{SD}_{8}$ & $\mathrm{~T}_{24}$ & $\mathrm{RH}_{8} \mathrm{RS}_{4} \mathrm{SD}_{8}$ \\
$\mathrm{~T}_{7}$ & $\mathrm{RH}_{0} \mathrm{RS}_{8} \mathrm{SD}_{0}$ & $\mathrm{~T}_{16}$ & $\mathrm{RH}_{4} \mathrm{RS}_{8} \mathrm{SD}_{0}$ & $\mathrm{~T}_{25}$ & $\mathrm{RH}_{8} \mathrm{RS}_{8} \mathrm{SD}_{0}$ \\
$\mathrm{~T}_{8}$ & $\mathrm{RH}_{0} \mathrm{RS}_{8} \mathrm{SD}_{4}$ & $\mathrm{~T}_{17}$ & $\mathrm{RH}_{4} \mathrm{RS}_{8} \mathrm{SD}_{4}$ & $\mathrm{~T}_{26}$ & $\mathrm{RH}_{8} \mathrm{RS}_{8} \mathrm{SD}_{4}$ \\
$\mathrm{~T}_{9}$ & $\mathrm{RH}_{0} \mathrm{RS}_{8} \mathrm{SD}_{8}$ & $\mathrm{~T}_{18} \mathrm{RH}_{4} \mathrm{RS}_{8} \mathrm{SD}_{8}$ & $\mathrm{~T}_{27}$ & $\mathrm{RH}_{8} \mathrm{RS}_{8} \mathrm{SD}_{8}$ \\
\hline
\end{tabular}

$\mathrm{RH}_{0} \mathrm{RH}_{4} \mathrm{RH}_{8}=$ Rice hull at the rate of $0,4 \& 8 \mathrm{tha}^{-1}$; RS0 RS4 RS8 $=$ Rice straw at the rate of 0,4 and $8 \mathrm{tha}^{-1}$; SD0 SD4 SD8 $=$ Sawdust at the rate of 0,4 and $8 \mathrm{tha}^{-1}$. 
potential of the soil to hold plant nutrients, by estimating the capacity of the soil to retain cations, which are positively-charged substances. Therefore, the CEC of the soil directly affects the amount and frequency of fertilizer application.

CEC of the post harvested soils were found to be increased with the application of rice hull, rice straw and sawdust under both the moisture levels in the pot and field experiments. The coefficient of variation $(\mathrm{CV})$ of different treatment means (Table III) indicate that the increment of CEC of the studied post harvested saline soils is significant $(p<0.001)$ except for the moist condition under field experiment. There was significant $(p<0.05)$ variation among the different treatments under pot experiment and in the field experiment under saturated soil condition.

In pot and field experiments, the highest (under pot exp.: 22.35 c mol kg-1@moist and 24.12 c mol kg-1@ saturated soil conditions; under field exp.:20.42 c mol kg-1@moist and $23.56 \mathrm{c}$ mol kg-1@ saturated soil conditions) and lowest values of CEC were obtained in the treatments $\mathrm{T}_{27}$ $\left(\mathrm{RH}_{8} \mathrm{RS}_{8} \mathrm{SD} 8\right)$ and $\mathrm{T}_{1}\left(\mathrm{RH}_{0} \mathrm{RS}_{0} \mathrm{SD}_{0}\right)$, respectively in post harvested soils (Table III). Although there was numerical variation in the CEC values in the two experiments, the trends of the increment and variation among different treatments were almost same. The results of the experiment revealed that the cation exchange capacity of

Table III. Post harvested soil CEC (c mol kg-1) values under pot (BRRI dhan 48) and field (BRRI dhan 64) experiments as influenced by the application of rice hull, rice straw, sawdust and moisture conditions

\begin{tabular}{|c|c|c|c|c|c|}
\hline \multicolumn{2}{|c|}{ Treatment } & \multicolumn{2}{|c|}{ BRRI dhan 48} & \multicolumn{2}{|c|}{ BRRI dhan 64} \\
\hline No. & Denotation & $\begin{array}{l}\text { Moist } \\
(80 \%)\end{array}$ & $\begin{array}{l}\text { Saturated } \\
(>100 \%)\end{array}$ & $\begin{array}{l}\text { Moist } \\
(80 \%)\end{array}$ & $\begin{array}{l}\text { Saturated } \\
(>100 \%)\end{array}$ \\
\hline $\mathrm{T}_{1}$ & $\mathrm{RH}_{0} \mathrm{RS}_{0} \mathrm{SD}_{0}$ & $17.38 \mathrm{~b}$ & $17.85 \mathrm{~b}$ & 17.64 & $17.69 \mathrm{~b}$ \\
\hline $\mathrm{T}_{2}$ & $\mathrm{RH}_{0} \mathrm{RS}_{0} \mathrm{SD}_{4}$ & $17.67 \mathrm{~b}$ & $17.89 \mathrm{~b}$ & 17.68 & $17.81 \mathrm{~b}$ \\
\hline $\mathrm{T}_{3}$ & $\mathrm{RH}_{0} \mathrm{RS}_{0} \mathrm{SD}_{8}$ & $17.69 \mathrm{~b}$ & $17.91 \mathrm{~b}$ & 17.69 & $17.83 \mathrm{bc}$ \\
\hline $\mathrm{T}_{4}$ & $\mathrm{RH}_{0} \mathrm{RS}_{4} \mathrm{SD}_{0}$ & $18.71 \mathrm{ab}$ & $18.89 \mathrm{ab}$ & 18.67 & $18.82 \mathrm{abc}$ \\
\hline $\mathrm{T}_{5}$ & $\mathrm{RH}_{0} \mathrm{RS}_{4} \mathrm{SD}_{4}$ & $18.72 \mathrm{ab}$ & $18.92 \mathrm{ab}$ & 18.71 & $18.90 \mathrm{abc}$ \\
\hline $\mathrm{T}_{6}$ & $\mathrm{RH}_{0} \mathrm{RS}_{4} \mathrm{SD}_{8}$ & $18.73 \mathrm{ab}$ & $19.08 \mathrm{ab}$ & 18.72 & $19.77 \mathrm{ac}$ \\
\hline $\mathrm{T}_{7}$ & $\mathrm{RH}_{0} \mathrm{RS}_{8} \mathrm{SD}_{0}$ & $18.72 \mathrm{ab}$ & $18.93 \mathrm{ab}$ & 18.69 & $18.95 \mathrm{abc}$ \\
\hline $\mathrm{T}_{8}$ & $\mathrm{RH}_{0} \mathrm{RS}_{8} \mathrm{SD}_{4}$ & $18.74 \mathrm{ab}$ & $19.91 \mathrm{ac}$ & 18.74 & $19.96 \mathrm{a}$ \\
\hline $\mathrm{T}_{9}$ & $\mathrm{RH}_{0} \mathrm{RS}_{8} \mathrm{SD}_{8}$ & $18.74 \mathrm{ab}$ & $19.96 \mathrm{ac}$ & 18.76 & $19.13 \mathrm{abc}$ \\
\hline $\mathrm{T}_{10}$ & $\mathrm{RH}_{4} \mathrm{RS}_{0} \mathrm{SD}_{0}$ & $19.68 \mathrm{ac}$ & $19.87 \mathrm{ac}$ & 19.69 & $19.78 \mathrm{ac}$ \\
\hline $\mathrm{T}_{11}$ & $\mathrm{RH}_{4} \mathrm{RS}_{0} \mathrm{SD}_{4}$ & $19.73 \mathrm{ac}$ & $19.92 \mathrm{ac}$ & 18.71 & $19.92 \mathrm{a}$ \\
\hline $\mathrm{T}_{12}$ & $\mathrm{RH}_{4} \mathrm{RS}_{0} \mathrm{SD}_{8}$ & $18.74 \mathrm{ab}$ & $19.97 \mathrm{ac}$ & 18.73 & $19.96 \mathrm{a}$ \\
\hline $\mathrm{T}_{13}$ & $\mathrm{RH}_{4} \mathrm{RS}_{4} \mathrm{SD}_{0}$ & $18.74 \mathrm{ab}$ & $20.07 \mathrm{ac}$ & 18.73 & $19.98 \mathrm{a}$ \\
\hline $\mathrm{T}_{14}$ & $\mathrm{RH}_{4} \mathrm{RS}_{4} \mathrm{SD}_{4}$ & $18.83 \mathrm{ab}$ & $20.17 \mathrm{ac}$ & 19.25 & $20.74 \mathrm{ad}$ \\
\hline $\mathrm{T}_{15}$ & $\mathrm{RH}_{4} \mathrm{RS}_{4} \mathrm{SD}_{8}$ & $20.87 \mathrm{cde}$ & $21.31 \mathrm{ce}$ & 19.57 & $20.78 \mathrm{ad}$ \\
\hline $\mathrm{T}_{16}$ & $\mathrm{RH}_{4} \mathrm{RS}_{8} \mathrm{SD}_{0}$ & $19.76 \mathrm{ac}$ & $20.15 \mathrm{ac}$ & 18.85 & $19.12 \mathrm{abc}$ \\
\hline $\mathrm{T}_{17}$ & $\mathrm{RH}_{4} \mathrm{RS}_{8} \mathrm{SD}_{4}$ & $19.92 \mathrm{ac}$ & 21.27 ce & 19.28 & $21.83 \mathrm{de}$ \\
\hline $\mathrm{T}_{18}$ & $\mathrm{RH}_{4} \mathrm{RS}_{8} \mathrm{SD}_{8}$ & $20.03 \mathrm{ac}$ & $22.34 \mathrm{de}$ & 19.34 & $21.97 \mathrm{de}$ \\
\hline $\mathrm{T}_{19}$ & $\mathrm{RH}_{8} \mathrm{RS}_{0} \mathrm{SD}_{0}$ & $18.69 \mathrm{ab}$ & $18.87 \mathrm{ab}$ & 18.72 & $18.91 \mathrm{abc}$ \\
\hline $\mathrm{T}_{20}$ & $\mathrm{RH}_{8} \mathrm{RS}_{0} \mathrm{SD}_{4}$ & $18.75 \mathrm{ab}$ & $18.95 \mathrm{ab}$ & 18.71 & $18.95 \mathrm{abc}$ \\
\hline $\mathrm{T}_{21}$ & $\mathrm{RH}_{8} \mathrm{RS}_{0} \mathrm{SD}_{8}$ & $18.79 \mathrm{ab}$ & $19.03 \mathrm{ab}$ & 18.73 & $19.07 \mathrm{abc}$ \\
\hline $\mathrm{T}_{22}$ & $\mathrm{RH}_{8} \mathrm{RS}_{4} \mathrm{SD}_{0}$ & $19.76 \mathrm{ac}$ & $19.21 \mathrm{ab}$ & 18.83 & $18.91 \mathrm{abc}$ \\
\hline $\mathrm{T}_{23}$ & $\mathrm{RH}_{8} \mathrm{RS}_{4} \mathrm{SD}_{4}$ & $21.84 \mathrm{de}$ & $22.34 \mathrm{de}$ & 19.26 & $21.84 \mathrm{de}$ \\
\hline $\mathrm{T}_{24}$ & $\mathrm{RH}_{8} \mathrm{RS}_{4} \mathrm{SD}_{8}$ & 20.92 cde & $23.47 \mathrm{~d}$ & 19.27 & $22.93 \mathrm{e}$ \\
\hline $\mathrm{T}_{25}$ & $\mathrm{RH}_{8} \mathrm{RS}_{8} \mathrm{SD}_{0}$ & $19.79 \mathrm{ac}$ & $20.25 \mathrm{ac}$ & 18.82 & $19.12 \mathrm{abc}$ \\
\hline $\mathrm{T}_{26}$ & $\mathrm{RH}_{8} \mathrm{RS}_{8} \mathrm{SD}_{4}$ & $21.18 \mathrm{cde}$ & $23.76 \mathrm{~d}$ & 19.29 & $22.15 \mathrm{de}$ \\
\hline $\mathrm{T}_{27}$ & $\mathrm{RH}_{8} \mathrm{RS}_{8} \mathrm{SD}_{8}$ & $22.35 \mathrm{e}$ & $24.12 \mathrm{~d}$ & 20.42 & $23.56 \mathrm{e}$ \\
\hline & $\mathrm{CV}$ & $4.47 * * *$ & $9.07 * * *$ & $1.06 \mathrm{NS}$ & $7.25 * * *$ \\
\hline
\end{tabular}

$\mathrm{CV}=$ Coefficient of Variation, $* * *$ indicates significant at $0.1 \%$ level, NS $=$ Not Significant. In a column, means followed by a common letter are not significantly different at $5 \%$ level by Tukey's Range Test. 
Table IV. Post harvested soil exchangeable cations $\left(\mathrm{Na}^{+}, \mathrm{K}^{+}, \mathrm{Ca}^{2+}\right.$ and $\mathrm{Mg}^{2+}$ ) values under pot (BRRI dhan 48) experiment as influenced by the application of rice hull, rice straw, sawdust and moisture conditions

\begin{tabular}{|c|c|c|c|c|c|c|c|c|c|}
\hline \multicolumn{2}{|c|}{ Treatment } & \multicolumn{2}{|c|}{$\mathrm{Na}^{+}\left(\mathrm{c} \mathrm{mol} \mathrm{kg}{ }^{-1}\right)$} & \multicolumn{2}{|c|}{$\mathrm{K}^{+}\left(\mathrm{c} \mathrm{mol} \mathrm{kg}{ }^{-1}\right)$} & \multicolumn{2}{|c|}{$\mathrm{Ca}^{2+}\left(\mathrm{c} \mathrm{mol} \mathrm{kg}^{-1}\right)$} & \multicolumn{2}{|c|}{$\mathrm{Mg}^{2+}\left(\mathrm{c} \mathrm{mol} \mathrm{kg}^{-1}\right)$} \\
\hline No. & Denotation & $\begin{array}{l}\text { Moist } \\
(80 \%)\end{array}$ & $\begin{array}{l}\text { Saturated } \\
(>100 \%)\end{array}$ & $\begin{array}{l}\text { Moist } \\
(80 \%)\end{array}$ & $\begin{array}{l}\text { Saturated } \\
(>100 \%)\end{array}$ & $\begin{array}{l}\text { Moist } \\
(80 \%)\end{array}$ & $\begin{array}{l}\text { Saturated } \\
(>100 \%)\end{array}$ & $\begin{array}{l}\text { Moist } \\
(80 \%)\end{array}$ & $\begin{array}{l}\text { Saturated } \\
(>100 \%)\end{array}$ \\
\hline $\mathrm{T}_{1}$ & $\mathrm{RH}_{0} \mathrm{RS}_{0} \mathrm{SD}_{0}$ & $3.64 \mathrm{a}$ & $3.61 \mathrm{a}$ & 0.13 & $0.14 \mathrm{a}$ & 0.74 & 0.85 & 3.25 & 3.25 \\
\hline $\mathrm{T}_{2}$ & $\mathrm{RH}_{0} \mathrm{RS}_{0} \mathrm{SD}_{4}$ & $3.63 \mathrm{a}$ & $3.60 \mathrm{a}$ & 0.14 & $0.19 \mathrm{ab}$ & 0.89 & 0.91 & 3.25 & 3.28 \\
\hline $\mathrm{T}_{3}$ & $\mathrm{RH}_{0} \mathrm{RS}_{0} \mathrm{SD}_{8}$ & $3.59 \mathrm{a}$ & $3.52 \mathrm{a}$ & 0.15 & $0.22 \mathrm{ab}$ & 0.87 & 0.89 & 3.27 & 3.35 \\
\hline $\mathrm{T}_{4}$ & $\mathrm{RH}_{0} \mathrm{RS}_{4} \mathrm{SD}_{0}$ & $3.61 \mathrm{a}$ & $3.53 \mathrm{a}$ & 0.19 & $0.23 \mathrm{ab}$ & 1.10 & 1.19 & 3.29 & 3.37 \\
\hline $\mathrm{T}_{5}$ & $\mathrm{RH}_{0} \mathrm{RS}_{4} \mathrm{SD}_{4}$ & $3.57 \mathrm{a}$ & $3.50 \mathrm{a}$ & 0.36 & $0.41 \mathrm{ab}$ & 1.26 & 1.93 & 3.31 & 3.38 \\
\hline $\mathrm{T}_{6}$ & $\mathrm{RH}_{0} \mathrm{RS}_{4} \mathrm{SD}_{8}$ & $3.54 \mathrm{a}$ & $3.51 \mathrm{a}$ & 0.37 & $0.42 \mathrm{ab}$ & 1.53 & 2.01 & 3.32 & 3.42 \\
\hline $\mathrm{T}_{7}$ & $\mathrm{RH}_{0} \mathrm{RS}_{8} \mathrm{SD}_{0}$ & $3.61 \mathrm{a}$ & $3.54 \mathrm{a}$ & 0.29 & $0.54 \mathrm{ab}$ & 1.77 & 1.61 & 3.26 & 3.39 \\
\hline $\mathrm{T}_{8}$ & $\mathrm{RH}_{0} \mathrm{RS}_{8} \mathrm{SD}_{4}$ & $3.58 \mathrm{a}$ & $3.56 \mathrm{a}$ & 0.49 & $0.66 \mathrm{ab}$ & 1.93 & 1.88 & 3.27 & 3.41 \\
\hline $\mathrm{T}_{9}$ & $\mathrm{RH}_{0} \mathrm{RS}_{8} \mathrm{SD}_{8}$ & $3.57 \mathrm{a}$ & $3.54 \mathrm{a}$ & 0.60 & $0.88 \mathrm{ab}$ & 2.05 & 2.82 & 3.29 & 3.45 \\
\hline $\mathrm{T}_{10}$ & $\mathrm{RH}_{4} \mathrm{RS}_{0} \mathrm{SD}_{0}$ & $3.63 \mathrm{a}$ & $3.60 \mathrm{a}$ & 0.64 & $0.65 \mathrm{ab}$ & 1.83 & 1.76 & 3.26 & 3.35 \\
\hline $\mathrm{T}_{11}$ & $\mathrm{RH}_{4} \mathrm{RS}_{0} \mathrm{SD}_{4}$ & $3.62 \mathrm{a}$ & $3.60 \mathrm{a}$ & 0.67 & $0.71 \mathrm{ab}$ & 1.89 & 1.83 & 3.26 & 3.38 \\
\hline $\mathrm{T}_{12}$ & $\mathrm{RH}_{4} \mathrm{RS}_{0} \mathrm{SD}_{8}$ & $3.61 \mathrm{a}$ & $3.56 \mathrm{a}$ & 0.68 & $0.74 \mathrm{ab}$ & 1.92 & 2.41 & 3.27 & 3.39 \\
\hline $\mathrm{T}_{13}$ & $\mathrm{RH}_{4} \mathrm{RS}_{4} \mathrm{SD}_{0}$ & $3.63 \mathrm{a}$ & $3.59 \mathrm{a}$ & 0.69 & $0.76 \mathrm{ab}$ & 1.79 & 1.74 & 3.26 & 3.39 \\
\hline $\mathrm{T}_{14}$ & $\mathrm{RH}_{4} \mathrm{RS}_{4} \mathrm{SD}_{4}$ & $2.56 \mathrm{ab}$ & $2.53 \mathrm{ab}$ & 1.71 & $1.78 \mathrm{abc}$ & 2.08 & 2.03 & 3.27 & 3.45 \\
\hline $\mathrm{T}_{15}$ & $\mathrm{RH}_{4} \mathrm{RS}_{4} \mathrm{SD}_{8}$ & $2.55 \mathrm{ab}$ & $2.54 \mathrm{ab}$ & 1.72 & $1.80 \mathrm{abc}$ & 1.89 & 1.81 & 3.28 & 3.48 \\
\hline $\mathrm{T}_{16}$ & $\mathrm{RH}_{4} \mathrm{RS}_{8} \mathrm{SD}_{0}$ & $3.62 \mathrm{a}$ & $3.57 \mathrm{a}$ & 0.69 & $0.78 \mathrm{ab}$ & 1.83 & 1.79 & 3.27 & 3.40 \\
\hline $\mathrm{T}_{17}$ & $\mathrm{RH}_{4} \mathrm{RS}_{8} \mathrm{SD}_{4}$ & $1.57 \mathrm{bc}$ & $1.52 \mathrm{bc}$ & 1.73 & $1.82 \mathrm{abc}$ & 1.86 & 1.84 & 3.27 & 3.46 \\
\hline $\mathrm{T}_{18}$ & $\mathrm{RH}_{4} \mathrm{RS}_{8} \mathrm{SD}_{8}$ & $3.53 \mathrm{a}$ & $3.51 \mathrm{a}$ & 1.73 & $2.18 b c$ & 1.89 & 1.91 & 3.29 & 3.47 \\
\hline $\mathrm{T}_{19}$ & $\mathrm{RH}_{8} \mathrm{RS}_{0} \mathrm{SD}_{0}$ & $3.64 \mathrm{a}$ & $3.60 \mathrm{a}$ & 0.64 & $0.69 \mathrm{ab}$ & 1.81 & 1.80 & 3.26 & 3.36 \\
\hline $\mathrm{T}_{20}$ & $\mathrm{RH}_{8} \mathrm{RS}_{0} \mathrm{SD}_{4}$ & $3.61 \mathrm{a}$ & $3.55 \mathrm{a}$ & 0.68 & $0.73 \mathrm{ab}$ & 1.78 & 1.77 & 3.27 & 3.39 \\
\hline $\mathrm{T}_{21}$ & $\mathrm{RH}_{8} \mathrm{RS}_{0} \mathrm{SD}_{8}$ & $3.61 \mathrm{a}$ & $3.53 \mathrm{a}$ & 0.68 & $0.75 \mathrm{ab}$ & 1.83 & 1.79 & 3.28 & 3.41 \\
\hline $\mathrm{T}_{22}$ & $\mathrm{RH}_{8} \mathrm{RS}_{4} \mathrm{SD}_{0}$ & $2.57 \mathrm{ab}$ & $2.45 \mathrm{ab}$ & 0.69 & $0.77 \mathrm{ab}$ & 1.81 & 1.79 & 3.26 & 3.39 \\
\hline $\mathrm{T}_{23}$ & $\mathrm{RH}_{8} \mathrm{RS}_{4} \mathrm{SD}_{4}$ & $1.56 \mathrm{bc}$ & $1.33 \mathrm{bc}$ & 1.72 & $2.78 \mathrm{c}$ & 1.91 & 1.78 & 3.27 & 3.46 \\
\hline $\mathrm{T}_{24}$ & $\mathrm{RH}_{8} \mathrm{RS}_{4} \mathrm{SD}_{8}$ & $1.53 \mathrm{bc}$ & $1.35 \mathrm{bc}$ & 1.73 & $2.81 \mathrm{c}$ & 1.95 & 2.83 & 3.29 & 3.47 \\
\hline $\mathrm{T}_{25}$ & $\mathrm{RH}_{8} \mathrm{RS}_{8} \mathrm{SD}_{0}$ & $2.51 \mathrm{ab}$ & $2.47 \mathrm{ab}$ & 0.70 & $0.75 \mathrm{ab}$ & 1.84 & 1.79 & 3.27 & 3.40 \\
\hline $\mathrm{T}_{26}$ & $\mathrm{RH}_{8} \mathrm{RS}_{8} \mathrm{SD}_{4}$ & $1.46 \mathrm{bc}$ & $1.42 \mathrm{bc}$ & 1.73 & $2.80 \mathrm{c}$ & 1.93 & 2.91 & 3.27 & 3.47 \\
\hline \multirow[t]{2}{*}{$\mathrm{T}_{27}$} & $\mathrm{RH}_{8} \mathrm{RS}_{8} \mathrm{SD}_{8}$ & $0.45 \mathrm{c}$ & $0.24 \mathrm{c}$ & 1.74 & $2.86 \mathrm{c}$ & 2.01 & 2.97 & 3.29 & 3.48 \\
\hline & $\mathrm{CV}$ & $2.60 * *$ & $2.81 * * *$ & $1.08 \mathrm{NS}$ & $2.37 * *$ & $0.43 \mathrm{NS}$ & $0.92 \mathrm{NS}$ & 0.00 & $0.01 \mathrm{NS}$ \\
\hline
\end{tabular}

$\mathrm{CV}=$ Coefficient of Variation, $* * *$ indicates significant at $0.1 \%$ level, $* *$ indicates significant at $1 \%$ level,

NS= Not Significant. In a column, means followed by a common letter are not significantly different at 5\% level by Tukey's Range Test.

soils increased with the increase rates of the treatments. Rice hull, rice straw and sawdust alone and their combination were found to be additive in increasing CEC of soils under both moist and saturated conditions in pot and field experiments. This might be due to the inherent consequences of organic amendments (rice hull, rice straw and sawdust) on soil exchangeable cations.

This result is quite similar with Chaganti et al. (2015) who said that the composts application increases soil CEC and decreases ESP values compared with the unfertilized control. According to Walker and Bernal (2008), the mature compost and manure application have a greater effect on the CEC increase and on SAR reduction.

\section{Dynamics in Exchangeable Cations}

The exchangeable cations especially $\mathrm{Na}^{+}$content decreased significantly $(\mathrm{p}<0.05)$ with the increased rates of rice hull, rice straw and sawdust. The maintenance of moisture conditions was also played a vital role in the decrement of the Na contents that might be due to the dilution effect of irrigated water resulting congenial atmosphere on soil-plant-water relationship. Application of rice hull, rice straw and sawdust alone and their combination was found to be more effective in decreasing $\mathrm{Na}^{+}$content in soil under saturated condition (Tables IV and V). 
The maximum and minimum values of $\mathrm{Na}^{+}$contents were obtained in the treatment $\mathrm{T}_{1}\left(\mathrm{RH}_{0} \mathrm{RS}_{0} \mathrm{SD}_{0}\right)$ and $\mathrm{T}_{27}\left(\mathrm{RH}_{8} \mathrm{RS}_{8}\right.$ $\mathrm{SD}_{8}$ ) under both pot and field experiments. Rice hull, rice straw and sawdust decreased $\mathrm{Na}^{+}$content at saturated condition due to improved soil physical condition and water movement and release of $\mathrm{Ca}^{2+}, \mathrm{Mg}^{2+}$ divalent cations where ion competition retarded $\mathrm{Na}$ content during decomposition thereby divalent cations occupy the exchange sites.

The exchangeable $\mathrm{K}^{+}$content of soil increased significantly $(p<0.05)$ with the higher moisture content. Rice hull, rice straw and sawdust alone and in combination also increased
$\mathrm{K}^{+}$content of the soils under both pot and field experiments (Tables IV and V). The exchangeable $\mathrm{Ca}^{2+}$ and $\mathrm{Mg}^{2+}$ contents of soil followed almost the similar trends and effects as that of $\mathrm{K}^{+}$exhibited (Tables IV and V).

The maximum and minimum values of $\mathrm{K}^{+}, \mathrm{Ca}^{2+}$ and $\mathrm{Mg}^{2+}$ contents of the soils were recorded in the treatment $\mathrm{T}_{27}$ $\left(\mathrm{RH}_{8} \mathrm{RS}_{8} \mathrm{SD}_{8}\right)$ and $\mathrm{T}_{1}\left(\mathrm{RH}_{0} \mathrm{RS}_{0} \mathrm{SD}_{0}\right)$ under both pot and field experiments. The results of the present investigation are in accordance with the findings of Kaniz and Khan (2013), except for $\mathrm{K}^{+}$content, which might be due to high

Table V. Post harvested soil exchangeable cations $\left(\mathrm{Na}^{+}, \mathrm{K}^{+}, \mathrm{Ca}^{2+}\right.$ and $\left.\mathrm{Mg}^{2+}\right)$ values under field (BRRI dhan 64) experiment as influenced by the application of rice hull, rice straw, sawdust and moisture conditions

\begin{tabular}{|c|c|c|c|c|c|c|c|c|c|}
\hline \multicolumn{2}{|c|}{ Treatment } & \multicolumn{2}{|c|}{$\mathrm{Na}^{+}\left(\mathrm{c} \mathrm{mol} \mathrm{kg}{ }^{-1}\right)$} & \multicolumn{2}{|c|}{$\mathrm{K}^{+}\left(\mathrm{c} \mathrm{mol} \mathrm{kg}{ }^{-1}\right)$} & \multicolumn{2}{|c|}{$\mathrm{Ca}^{2+}\left(\mathrm{c} \mathrm{mol} \mathrm{kg}{ }^{-1}\right)$} & \multicolumn{2}{|c|}{$\mathrm{Mg}^{2+}\left(\mathrm{c} \mathrm{mol} \mathrm{kg}{ }^{-1}\right)$} \\
\hline No. & Denotation & $\begin{array}{l}\text { Moist } \\
(80 \%)\end{array}$ & $\begin{array}{l}\text { Saturated } \\
(>100 \%)\end{array}$ & $\begin{array}{l}\text { Moist } \\
(80 \%)\end{array}$ & $\begin{array}{l}\text { Saturated } \\
(>100 \%)\end{array}$ & $\begin{array}{l}\text { Moist } \\
(80 \%)\end{array}$ & $\begin{array}{l}\text { Saturated } \\
(>100 \%)\end{array}$ & $\begin{array}{l}\text { Moist } \\
(80 \%)\end{array}$ & $\begin{array}{l}\text { Saturated } \\
(>100 \%)\end{array}$ \\
\hline $\mathrm{T}_{1}$ & $\mathrm{RH}_{0} \mathrm{RS}_{0} \mathrm{SD}_{0}$ & $3.58 \mathrm{a}$ & $3.56 \mathrm{a}$ & 0.14 & $0.17 \mathrm{a}$ & 0.62 & 0.76 & 3.23 & 3.24 \\
\hline $\mathrm{T}_{2}$ & $\mathrm{RH}_{0} \mathrm{RS}_{0} \mathrm{SD}_{4}$ & $3.55 \mathrm{a}$ & $3.54 \mathrm{a}$ & 0.15 & $0.23 \mathrm{a}$ & 0.87 & 0.79 & 3.24 & 3.29 \\
\hline $\mathrm{T}_{3}$ & $\mathrm{RH}_{0} \mathrm{RS}_{0} \mathrm{SD}_{8}$ & $3.54 \mathrm{a}$ & $3.51 \mathrm{a}$ & 0.16 & $0.25 \mathrm{a}$ & 0.85 & 0.81 & 3.25 & 3.33 \\
\hline $\mathrm{T}_{4}$ & $\mathrm{RH}_{0} \mathrm{RS}_{4} \mathrm{SD}_{0}$ & $3.57 \mathrm{a}$ & $3.53 \mathrm{a}$ & 0.16 & $0.32 \mathrm{a}$ & 1.08 & 1.15 & 3.24 & 3.29 \\
\hline $\mathrm{T}_{5}$ & $\mathrm{RH}_{0} \mathrm{RS}_{4} \mathrm{SD}_{4}$ & $3.54 \mathrm{a}$ & $3.53 \mathrm{a}$ & 0.27 & $0.39 \mathrm{a}$ & 1.04 & 1.91 & 3.26 & 3.31 \\
\hline $\mathrm{T}_{6}$ & $\mathrm{RH}_{0} \mathrm{RS}_{4} \mathrm{SD}_{8}$ & $3.51 \mathrm{a}$ & $3.49 \mathrm{a}$ & 0.28 & $0.41 \mathrm{a}$ & 1.21 & 1.98 & 3.27 & 3.35 \\
\hline $\mathrm{T}_{7}$ & $\mathrm{RH}_{0} \mathrm{RS}_{8} \mathrm{SD}_{0}$ & $3.54 \mathrm{a}$ & $3.53 \mathrm{a}$ & 0.25 & $0.51 \mathrm{a}$ & 1.74 & 1.79 & 3.24 & 3.29 \\
\hline $\mathrm{T}_{8}$ & $\mathrm{RH}_{0} \mathrm{RS}_{8} \mathrm{SD}_{4}$ & $3.53 \mathrm{a}$ & $3.52 \mathrm{a}$ & 0.48 & $0.62 \mathrm{ab}$ & 1.91 & 1.86 & 3.27 & 3.33 \\
\hline $\mathrm{T}_{9}$ & $\mathrm{RH}_{0} \mathrm{RS}_{8} \mathrm{SD}_{8}$ & $3.53 \mathrm{a}$ & $3.51 \mathrm{a}$ & 0.69 & $0.81 \mathrm{ab}$ & 1.94 & 2.79 & 3.28 & 3.36 \\
\hline $\mathrm{T}_{10}$ & $\mathrm{RH}_{4} \mathrm{RS}_{0} \mathrm{SD}_{0}$ & $3.57 \mathrm{a}$ & $3.55 \mathrm{a}$ & 0.64 & $0.68 \mathrm{ab}$ & 1.82 & 1.74 & 3.24 & 3.26 \\
\hline $\mathrm{T}_{11}$ & $\mathrm{RH}_{4} \mathrm{RS}_{0} \mathrm{SD}_{4}$ & $3.56 \mathrm{a}$ & $3.53 \mathrm{a}$ & 0.65 & $0.73 \mathrm{ab}$ & 1.87 & 1.79 & 3.25 & 3.29 \\
\hline $\mathrm{T}_{12}$ & $\mathrm{RH}_{4} \mathrm{RS}_{0} \mathrm{SD}_{8}$ & $3.54 \mathrm{a}$ & $3.51 \mathrm{a}$ & 0.66 & $0.75 \mathrm{ab}$ & 1.89 & 2.57 & 3.26 & 3.31 \\
\hline $\mathrm{T}_{13}$ & $\mathrm{RH}_{4} \mathrm{RS}_{4} \mathrm{SD}_{0}$ & $3.54 \mathrm{a}$ & $3.53 \mathrm{a}$ & 0.67 & $0.73 \mathrm{ab}$ & 1.76 & 1.73 & 3.24 & 3.30 \\
\hline $\mathrm{T}_{14}$ & $\mathrm{RH}_{4} \mathrm{RS}_{4} \mathrm{SD}_{4}$ & $2.51 \mathrm{ab}$ & $2.49 \mathrm{ab}$ & 1.69 & $1.81 \mathrm{abc}$ & 2.04 & 2.01 & 3.29 & 3.32 \\
\hline $\mathrm{T}_{15}$ & $\mathrm{RH}_{4} \mathrm{RS}_{4} \mathrm{SD}_{8}$ & $2.49 \mathrm{ab}$ & $2.48 \mathrm{ab}$ & 1.69 & $1.83 \mathrm{abc}$ & 1.88 & 1.85 & 3.30 & 3.36 \\
\hline $\mathrm{T}_{16}$ & $\mathrm{RH}_{4} \mathrm{RS}_{8} \mathrm{SD}_{0}$ & $3.51 \mathrm{a}$ & $3.45 \mathrm{a}$ & 0.69 & $0.75 \mathrm{ab}$ & 1.79 & 1.76 & 3.27 & 3.31 \\
\hline $\mathrm{T}_{17}$ & $\mathrm{RH}_{4} \mathrm{RS}_{8} \mathrm{SD}_{4}$ & $1.47 \mathrm{bc}$ & $1.48 \mathrm{bc}$ & 1.68 & $1.82 \mathrm{abc}$ & 1.85 & 1.81 & 3.30 & 3.33 \\
\hline $\mathrm{T}_{18}$ & $\mathrm{RH}_{4} \mathrm{RS}_{8} \mathrm{SD}_{8}$ & $3.46 \mathrm{a}$ & $3.43 \mathrm{a}$ & 1.70 & $2.58 \mathrm{bc}$ & 1.87 & 1.85 & 3.30 & 3.35 \\
\hline $\mathrm{T}_{19}$ & $\mathrm{RH}_{8} \mathrm{RS}_{0} \mathrm{SD}_{0}$ & $3.53 \mathrm{a}$ & $3.47 \mathrm{a}$ & 0.65 & $0.69 \mathrm{ab}$ & 1.79 & 1.76 & 3.26 & 3.29 \\
\hline $\mathrm{T}_{20}$ & $\mathrm{RH}_{8} \mathrm{RS}_{0} \mathrm{SD}_{4}$ & $3.51 \mathrm{a}$ & $3.45 \mathrm{a}$ & 0.66 & $0.76 \mathrm{ab}$ & 1.77 & 1.73 & 3.27 & 3.31 \\
\hline $\mathrm{T}_{21}$ & $\mathrm{RH}_{8} \mathrm{RS}_{0} \mathrm{SD}_{8}$ & $3.51 \mathrm{a}$ & $3.49 \mathrm{a}$ & 0.67 & $0.79 \mathrm{ab}$ & 1.81 & 1.77 & 3.28 & 3.34 \\
\hline $\mathrm{T}_{22}$ & $\mathrm{RH}_{8} \mathrm{RS}_{4} \mathrm{SD}_{0}$ & $2.49 \mathrm{ab}$ & $2.45 \mathrm{ab}$ & 0.67 & $0.76 \mathrm{ab}$ & 1.76 & 1.75 & 3.25 & 3.32 \\
\hline $\mathrm{T}_{23}$ & $\mathrm{RH}_{8} \mathrm{RS}_{4} \mathrm{SD}_{4}$ & $1.47 \mathrm{bc}$ & $1.39 \mathrm{bc}$ & 1.69 & $2.82 \mathrm{c}$ & 1.88 & 1.75 & 3.30 & 3.36 \\
\hline $\mathrm{T}_{24}$ & $\mathrm{RH}_{8} \mathrm{RS}_{4} \mathrm{SD}_{8}$ & $1.45 \mathrm{bc}$ & $1.38 \mathrm{bc}$ & 1.71 & $2.84 \mathrm{c}$ & 1.89 & 2.80 & 3.29 & 3.37 \\
\hline $\mathrm{T}_{25}$ & $\mathrm{RH}_{8} \mathrm{RS}_{8} \mathrm{SD}_{0}$ & $2.49 \mathrm{ab}$ & $2.41 \mathrm{ab}$ & 0.69 & $0.78 \mathrm{ab}$ & 1.81 & 1.76 & 3.26 & 3.33 \\
\hline $\mathrm{T}_{26}$ & $\mathrm{RH}_{8} \mathrm{RS}_{8} \mathrm{SD}_{4}$ & $1.45 \mathrm{bc}$ & $1.27 \mathrm{bc}$ & 1.72 & $2.82 \mathrm{c}$ & 1.85 & 2.83 & 3.31 & 3.37 \\
\hline $\mathrm{T}_{27}$ & $\mathrm{RH}_{8} \mathrm{RS}_{8} \mathrm{SD}_{8}$ & $0.43 \mathrm{c}$ & $0.13 \mathrm{c}$ & 1.74 & $2.86 \mathrm{c}$ & 1.91 & 2.87 & 3.34 & 3.45 \\
\hline & $\mathrm{CV}$ & $2.56 * *$ & $2.81 * * *$ & $1.07 \mathrm{NS}$ & $2.50 * *$ & $0.48 \mathrm{NS}$ & $0.96 \mathrm{NS}$ & 0.00 & $0.01 \mathrm{NS}$ \\
\hline
\end{tabular}

$\mathrm{CV}=$ Coefficient of Variation, *** indicates significant at $0.1 \%$ level, ** indicates significant at $1 \%$ level,

$\mathrm{NS}=$ Not Significant. In a column, means followed by a common letter are not significantly different at $5 \%$ level by Tukey's Range Test 
bulk density and increased water movement created by them and release of $\mathrm{Ca}^{2+}, \mathrm{Mg}^{2+}$ divalent cations during decomposition of those indigenous organic materials. Combined application of rice hull, rice straw and sawdust were better than their individual application in increasing exchangeable potassium, calcium and magnesium status of the soils. These findings are almost similar with Oo et al. (2015), who revealed that organic amendment like compost is a source of nutrients, cations (in particular $\mathrm{Ca}$ and $\mathrm{K}$ ) and $\mathrm{C}$ that have an important role to promote the replacement of $\mathrm{Na}$ on exchange sites and increase microbial activity.

To reclaim effectively sodic and saline-sodic soils, inorganic amendments (gypsum, calcite, calcium chloride, and other chemical agents) are notoriously used as important sources of calcium to replace exchangeable $\mathrm{Na}$ on the exchange sites (Hanay et al., 2004). In this context, the application of organic amendments can represent an important and effective practice to maintain an appropriate soil structure, supply a sufficiently available cation amount (such as $\mathrm{Ca}, \mathrm{Mg}, \mathrm{K}$, etc.) and increase the fertility of saline and/or sodic soils. In fact, the organic matter plays a fundamental role on the structural stability of soil aggregate (Chaganti et al., 2015) by binding of mineral particles to organic polymers.

Ranjbar and Jalali (2011) used leaching columns and studied the effects of several plant residues (wheat, potato, sunflower, and rape) added to a sandy loam soil, irrigated with water having three different sodium adsorption ratios. In a similar experiment, Jalali and Ranjbar (2009) evaluated the effects of poultry and sheep manure. In both leaching experiments, conducted under saturated conditions at room
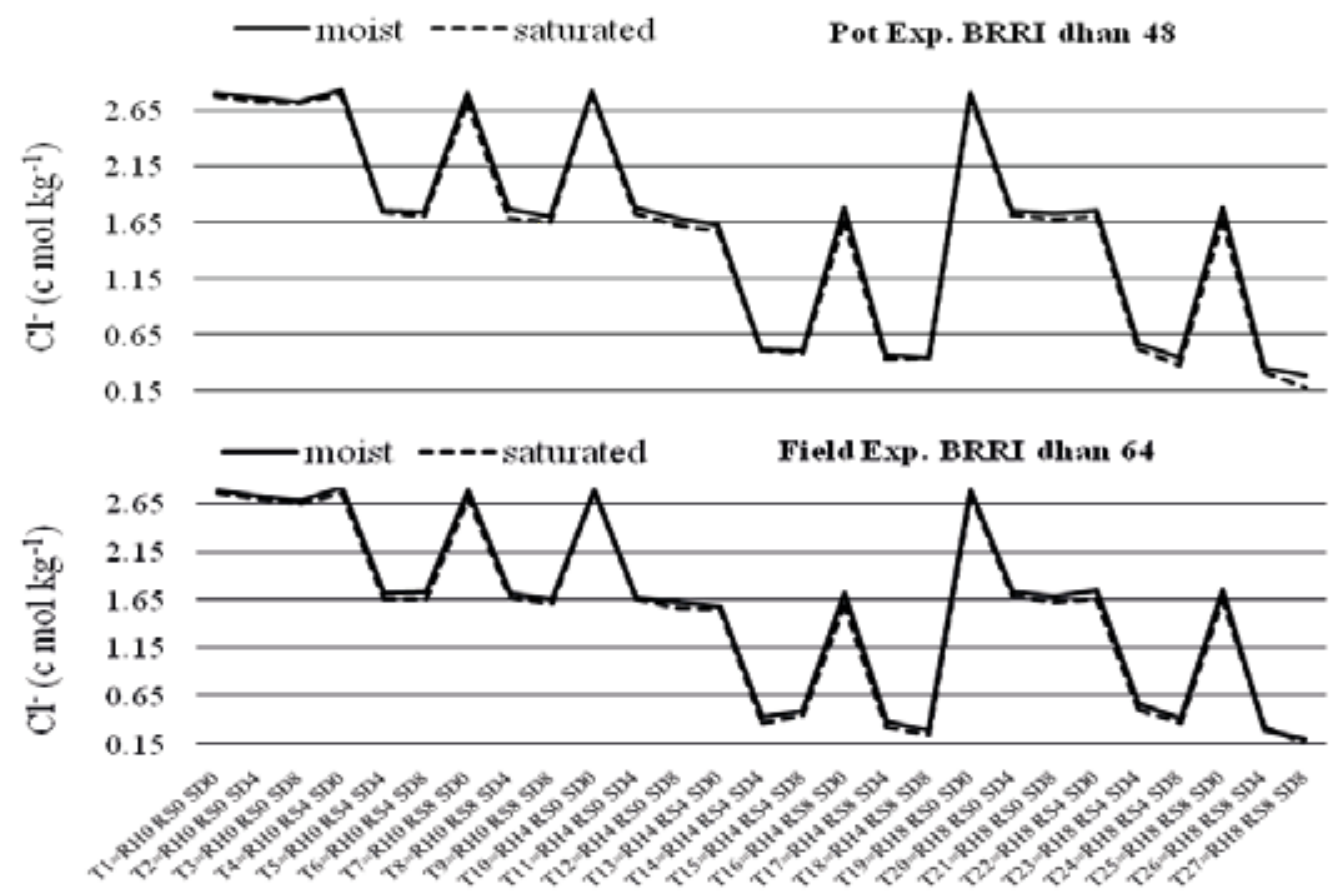

Treatment

\begin{tabular}{cccc}
\hline & Rice cultivar & Moisture level & $\mathrm{CV}$ \\
\hline & BRRI dhan 48 & $80 \%$ & $2.40^{* *}$ \\
$\mathrm{Cl}^{-}$ & & $>100 \%$ & $2.42^{* *}$ \\
$\left(\mathrm{c} \mathrm{mol} \mathrm{kg}{ }^{-1}\right)$ & BRRI dhan 64 & $80 \%$ & $2.49^{* *}$ \\
& & $>100 \%$ & $2.48^{* *}$ \\
\hline
\end{tabular}

Fig. 1. Contents of $\mathrm{Cl}^{-}\left(\mathrm{c} \mathrm{mol} \mathrm{kg}{ }^{-1}\right)$ at post harvest soils under pot (BRRI dhan 48) and field (BRRI dhan 64) experiments as influenced by the application of rice hull, rice straw, sawdust and moisture conditions 
temperature, the organic material applied was the same and the results indicated that the application of organic amendments to soils caused an increase in CEC compared with unamended control and a greater adsorption of $\mathrm{Ca}^{2+}$, $\mathrm{Mg}^{2+}$, and $\mathrm{K}^{+}$at the expense of $\mathrm{Na}^{+}$.

\section{Dynamics in Anions}

Among the water-soluble anions $\left(\mathrm{Cl}^{-}, \mathrm{SO}_{4}{ }^{2-}\right.$ and $\left.\mathrm{HCO}_{3}^{-}\right)$, chloride $\left(\mathrm{Cl}^{-}\right)$was dominant and significantly decreased with irrigation under both pot $(\mathrm{CV}=2.40 * * @$ moist, $2.42 * * @$ saturated condition) and field (CV=2.49**@ moist,2.48**@saturated condition) experiments. Rice hull, rice straw and sawdust alone and their combination were found to be significant $(\mathrm{p}<0.05)$ in decreasing $\mathrm{Cl}^{-}$ ions in post harvested soils (Fig. 1).

The highest and lowest $\mathrm{Cl}^{-}$contents were evaluated in the treatment $\mathrm{T}_{1}\left(\mathrm{RH}_{0} \mathrm{RS}_{0} \mathrm{SD}_{0}\right)$ and $\mathrm{T}_{27}\left(\begin{array}{llll}\mathrm{RH}_{8} & \mathrm{RS}_{8} & \mathrm{SD}_{8}\end{array}\right)$, respectively for all the varieties tested.

Sulfate content of post harvested soil increased with the application of rice hull, rice straw, sawdust and moisture conditions (Fig. 2). The higher and lower values were recorded in $\mathrm{T}_{27}\left(\mathrm{RH}_{8} \mathrm{RS}_{8} \mathrm{SD}_{8}\right)$ and $\mathrm{T}_{1}\left(\mathrm{RH}_{0} \mathrm{RS}_{0} \mathrm{SD}_{0}\right)$ treatments under both pot and field experiments. Bicarbonate $\left(\mathrm{HCO}_{3}^{-}\right)$ contents of the post harvested soils also showed almost similar trend as those shown by sulfate contents (Fig. 3). Individual application of rice straw and saw dust were found better in increasing sulfate and bicarbonate contents while decrease chloride contents than that of rice hull treatment but their combined application was found more additive than that of their single dosage.

\section{Conclusion}

The present findings concluded that the application of rice hull, rice straw and sawdust as organic amendments were found to be increased CEC, exchangeable $\mathrm{K}^{+}, \mathrm{Ca}^{2+}, \mathrm{Mg}^{2+}$ and decreased $\mathrm{Na}^{+}$contents of the studied saline soils. Among the water-soluble anions, $\mathrm{Cl}^{-}$content was decreased, while $\mathrm{SO}_{4}{ }^{2-}$ and $\mathrm{HCO}_{3}$-contents were increased by the application of these organic amendments under both moisture levels as maintained at the pot and field experiments. The combined application of rice hull, rice straw and sawdust each at the rate of $8 \mathrm{t} \mathrm{ha}^{-1}$ under saturated condition was attained the best performance among the treatments, which reveals that the interactions among these organic amendments were synergistic and would be effective in order to boost up the saline soil properties.

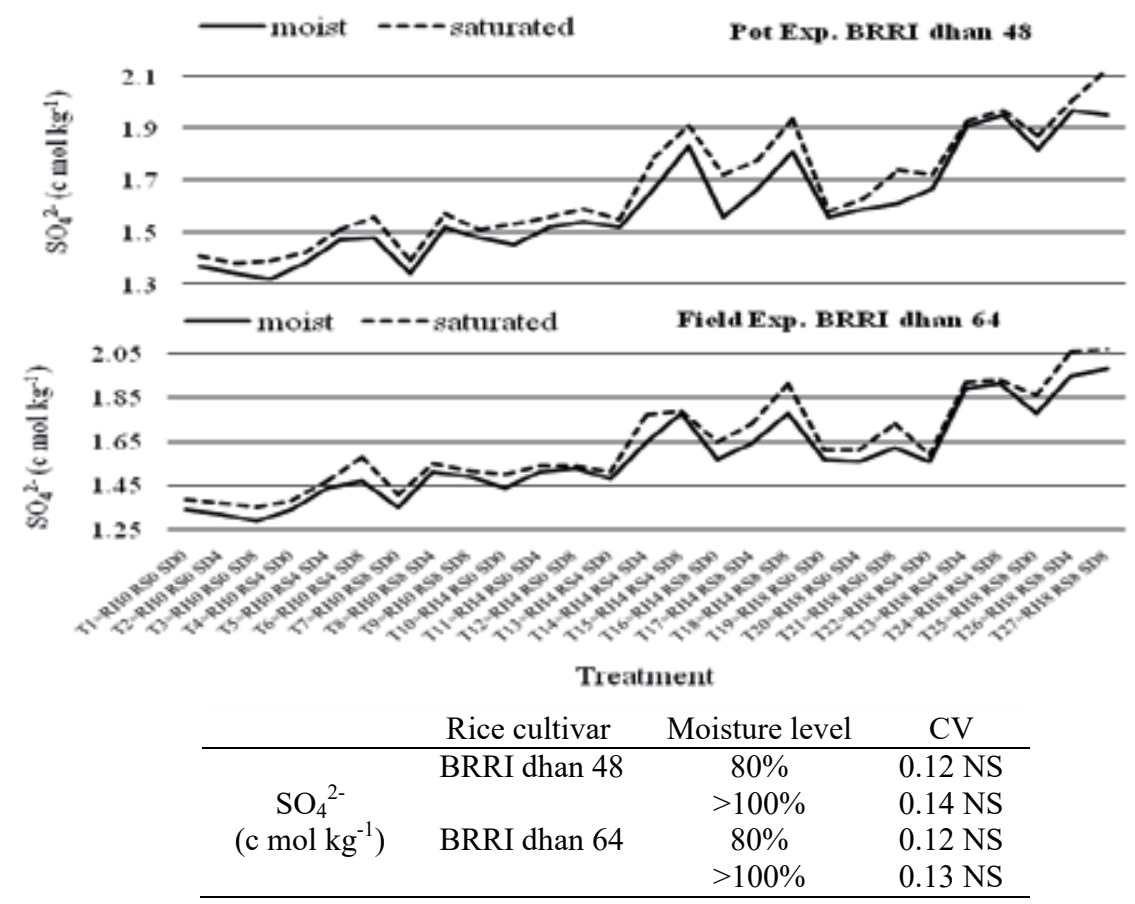

Fig. 2. Contents of $\mathrm{SO}_{4}{ }^{2-}\left(\mathrm{c} \mathrm{mol} \mathrm{kg}^{-1}\right)$ at post harvest soils under pot (BRRI dhan 48) and field (BRRI dhan 64) experiments as influenced by the application of rice hull, rice straw, sawdust and moisture conditions 


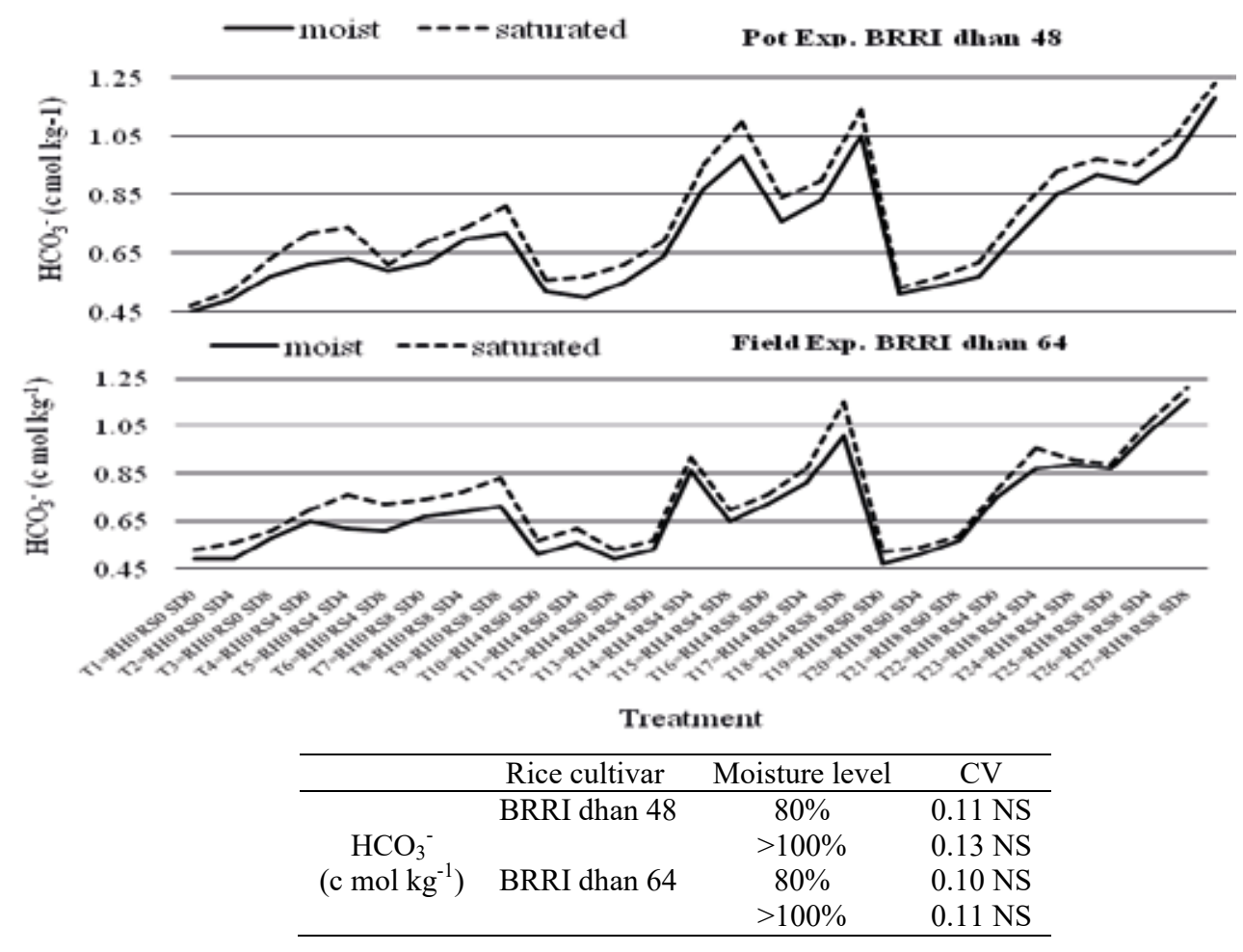

Fig. 3. Contents of $\mathrm{HCO}_{3}^{-}\left(\mathrm{c} \mathrm{mol} \mathrm{kg} \mathrm{g}^{-1}\right)$ at post harvest soils under pot (BRRI dhan 48) and field (BRRI dhan $64^{3}$ ) experiments as influenced by the application of rice hull, rice straw, sawdust and moisture conditions

\section{Acknowledgement}

The study was carried out under the project entitled 'Assessment of Impacts of Climate Change on Soil Health and Food Security, and Adaptation of Climate-smart Agriculture in Most Adversely Affected Areas of Bangladesh' funded by BCCT (2017-20), MoEFCC, Government of the Peoples' Republic of Bangladesh. Ministry of Science and Technology is gratefully acknowledged for providing NST fellowship for the first author.

\section{References}

Akter S, Khan HR and Hossain MS (2017), Potentials of rice straw, rice hull and saw dust on the growth and yield performance of rice grown under variable moisture conditions in saline soil, $J$ Asiat Soc Bangladesh Sci. 43(2): 197-206. DOI: org/10.3329/jasbs.v43i2.46517
Black CA (1965), Methods of soil analysis. Part 2. Am soc. Agron. Inst. Publ., Madison, Wisconsin, USA, pp 894-1372.

Chaganti VN, Crohn DM and Simłnek J (2015), Leaching and reclamation of a biochar and compost amended saline-sodic soil with moderate SAR reclaimed water, Agricultural Water Management. 158: 255-65. DOI: org/10.1016/j.agwat.2015.05.016

Gupta SK and Gupta IC (1987), Management of Saline Soils and Waters. Oxford and IBH Publication, New Delhi.

Hanay A, Büyüksönmez F, Kiziloglu FM and Canbolat MY (2004), Reclamation of saline- sodic soils with gypsum and MSW compost, Compost Science and Utilization. 12(2): 175-179. DOI: 10.1080/1065657X.2004. 10702177 
Islam MR (2006), Managing diverse land uses in coastal Bangladesh: institutional approaches In: Environment and livelihoods in tropical coastal zones, Eds. Hoanh CT, Tuong TP, Gowing JW and Hardy B, CAB International 38: 237-248,

Jackson ML (1973), Soil chemical analysis. Prentice hall of India Pvt. Ltd. New Delhi, India, pp 36-196.

Jalali M and Ranjbar F (2009), Effects of sodic water on soil sodicity and nutrient leaching in poultry and sheep manure amended soils, Geoderma. 153(1-2): 194-204.

DOI: org/10.1016/j.geoderma.2009.08.004

Julkowska MM and Testerink C (2015), Tuning plant signaling and growth to survive salt, Trends Plant Sci. 20(9): 586-594. DOI: org/10.1016/ j.tplants. 2015.06.008.

Kaniz F and Khan HR (2013), Reclamation of saline soil using gypsum, rice hull and sawdust in relation to rice production, $J$ Adv Sci Res. 4(3): 1-5.

Munns R, James RA, Gilliham M, Flowers TJ and Colmer TD (2016), Tissue tolerance:an essential but elusive trait for salt-tolerant crops, Funct. Plant Biol. 43(12): 1103-1113.

Munns R, Passioura JB, Colmer TD and Byrt CS (2020), Osmotic adjustment and energy limitations to plant growth in saline soil, New Phytol. 225(3): 1091-1096. DOI: org/10.1111/nph.15862.

Nelson DW and Sommers LE (1982), Total carbon, organic carbon and organic matter In: Methods of soil analysis, Ed. Page AL, part 2, Am. Soc. Agron. Inst. Publ., Madison, WI, USA, pp 539-579.

Olsen SR, Cale CV, Watanabe FS and Dean LA (1954), Estimation of available phosphorus in soils by extraction with sodium bicarbonate, USDA Circ. 939.

OoAN, Iwai CB and Saenjan P (2015), Soil properties and maize growth in saline and nonsaline soils using cassava-industrial waste compost and vermicompost with and without earthworms, Land Degradation and Development 26: 300-310. DOI: 10.1002/Idr.2208
Oorts K, Vanlauwe B and Merckx R (2003), Cation exchange capacities of soil organic matter fractions in a ferric lixisol with different organic matter inputs. Agriculture, Ecosystems and Environment 100(2-3): 161-71.

Piper CS (1966), Soil and plant analysis, Hano Publ. Bombay, India.

Pratt PF (1965), Methods of Soil Analysis, Am. Soc. Agron. Madison, Wisconsin, USA, p 1022.

Raafat NZ and Tharwat EER (2011), Improving wheat grain yield and its quality under salinity conditions at a newly reclaimed soil using different organic sources as soil or foliar applications, J Appl Sci Res. 7: $42-55$.

Rahman MA, Hassan KM, Alam M, Akid ASM and Riyad ASM (2014), Effects of salinity on land fertility in coastal areas of Bangladesh, International Journal of Renewable Energy and Environmental Engineering 2(3): 174-179.

Ranjbar F and Jalali M (2011), Effects of plant residues and calcite amendments on soil sodicity, Journal of Plant Nutrition and Soil Science 174(6): 874-83. DOI: org/10.1002/jpln.201000247

Richards LA (1954), Diagnosis and improvement of saline and alkali soils In: USDA Handbook No. 60, US Govt. Print. Office, Washington, USA, pp 84-156.

Seleiman MF and Kheir AMS (2018), Saline soil properties, quality and productivity of wheat grown with bagasse ash and thiourea in different climatic zones, Chemosphere 193: 538-546. DOI: 10.1016/j. chemosphere.2017.11.053

Walker DJ and Bernal MP (2008), The effects of olive mill waste compost and poultry manure on the availability and plant uptake of nutrients in a highly saline soil, Bioresource Technology 99(2): 396-403. DOI: org/10.1016/j.biortech.2006.12.006

Yupeng W, Yufei L, Yi Z, Yanmeng B and Zhenjun S (2018), Responses of Saline Soil Properties and Cotton Growth to Different Organic Amendments, Pedosphere 28: 521-529. DOI: org/10.1016 /S1002-0160(17)60464-8 